\title{
CONSERVAÇÃO DE SEMENTE DE Cedrela fissilis: TEOR DE ÁGUA DA SEMENTE E TEMPERATURA DO AMBIENTE ${ }^{1}$
}

\author{
LEILA MARTINS ${ }^{2}$, ANTONIO AUGUSTO DO LAGO ${ }^{3}$
}

\begin{abstract}
RESUMO - Foi realizado o estudo do comportamento fisiológico da semente de cedro-rosa (Cedrela fissilis Vellozo), em relação a variações do grau de umidade da semente e da temperatura do ambiente, durante o armazenamento. Frutos colhidos manualmente de plantas-matrizes foram colocados em ambiente sombreado, para posterior extração da semente. Foi determinado o grau de umidade inicial do lote e, paralelamente, obtida a amostra representante dos tratamentos, com o maior teor de água a ser estudado (16,3\%). As sementes remanescentes foram submetidas a secagem, em secador com circulação forçada de ar a $30^{\circ} \mathrm{C}$, para a obtenção dos demais graus de umidade desejados $(12,4$ \%, $8,1 \%$ e $4,5 \%$ ). As amostras, correspondentes aos diferentes graus de umidade, foram armazenadas em câmaras com temperaturas de $10^{\circ}$ e $20^{\circ} \mathrm{C}$. No início e após 40, 120, 200, 280 e 360 dias de armazenamento, a semente foi submetida às seguintes avaliações fisiológicas: germinação, emergência e comprimento da parte aérea. O delineamento experimental foi o inteiramente casualizado, em esquema fatorial envolvendo quatro tratamentos (graus de umidade) no início do armazenamento e oito tratamentos (quatro graus de umidade $\mathrm{x}$ duas condições térmicas), em cada época de avaliação, durante o armazenamento. A comparação das médias foi realizada pelo teste de Tukey, ao nível de 5 $\%$. A conservação de semente de cedro, considerando o intervalo de $16,3 \%$ a 4,5 \%, para o teor de água, é favorecida pelos graus de umidade entre $12,4 \%$ e $4,5 \%$, nas temperaturas de $20^{\circ}$ e $10^{\circ} \mathrm{C}$.
\end{abstract}

Termos para indexação: cedro-rosa, condição de armazenamento, qualidade fisiológica.

\section{Cedrela fissilis SEED PRESERVATION: SEED WATER CONTENT AND AMBIENT TEMPERATURE}

\begin{abstract}
The objective of this research was to study the physiological performance of Cedrela fissilis Vellozo seeds during storage in relation to variations in seed moisture content and storage temperature. Mature fruits were hand harvested from selected trees and placed under shade to complete the drying process to allow easy seed extraction from the naturally opened fruits. The initial moisture content of the seed lot was determined and a sample was drawn to represent the highest moisture content to be studied (16,3\%). Following that, the remaining seeds were dried in a forced air dryer at $30^{\circ} \mathrm{C}$ to obtain the other desired moisture contents $(12.4 \%, 8.1 \%$ and $4.5 \%)$. The samples corresponding to the different levels of moisture were stored inside chambers set at 10 and $20^{\circ} \mathrm{C}$. At the beginning and at 40,120, 200, 280, and 360 days of storage, seeds were submitted to the following physiological evaluations: germination, emergence and hypocotyl length. A $4 \times 2$ factorial in a completely random design was used involving four treatments (moisture contents) at the
\end{abstract}

${ }^{1}$ Submetido em 15/02/2007. Aceito para publicação em 28/12/2007. Apoiado financeiramente pela Fundação de Amparo à Pesquisa do Estado de São Paulo (FAPESP).

${ }^{2}$ Bolsista do CNPq, Laboratório Central de Sementes e Mudas, Departamento de Sementes Mudas e Matrizes - CATI. Caixa Postal 962, CEP 13070-178, Campinas, SP, Brasil, e-mail: leila@cati.sp.gov.br

${ }^{3}$ Instituto Agronômico de Campinas/APTA. Caixa Postal 28, CEP $13012-$ 970, Campinas, SP, Brasil, email: aalago@iac.sp.gov.br 
beginning of storage, and eight treatments (4 moisture contents $\mathrm{x} 2$ thermal conditions) at all other storage times. Mean comparison was made by the Tukey test at $5 \%$. Cedrela fissilis seed preservation, considering the $16.3 \%$ to $4.5 \%$ moisture content interval, is favored by moisture contents between $12.4 \%$ and $4.5 \%$, at temperatures of 20 and $10^{\circ} \mathrm{C}$.

Index terms: wood species, storage condition, physiological quality.

\section{INTRODUÇÃO}

Os tecnologistas de semente florestal encontram ainda dificuldades no estabelecimento de técnicas de produção, manipulação e avaliação da qualidade dessa semente. Os problemas decorrem da diversidade morfológica dos frutos e da dificuldade de extração da semente. Adicionalmente, a produção aleatória de frutos, de um ano para outro, pode prejudicar a formação de estoques reguladores e, também, proporcionar lotes com quantidade insuficiente de semente para os testes de avaliação da qualidade (Aguiar et al., 1993). Além disso, as informações são escassas para a definição de procedimentos capazes de ampliar o período de conservação da semente, já que o conhecimento atual é limitado, concentrado, principalmente, em plantas de interesse agrícola.

Apesar do aumento considerável dos estudos com semente de espécies florestais nativas, ainda há carência de informações básicas sobre muitas espécies como, por exemplo, o cedro-rosa (Cedrela fissilis Vellozo), de alto valor econômico e paisagístico.

Cedrela fissilis Vellozo, conhecida popularmente como cedro-rosa ou cedro-branco, éuma Meliaceae de porte arbóreo, com características de planta parcialmente umbrófila, no estádio juvenil, e heliófila, no estádio adulto. Por apresentar maior desenvolvimento sob condição menos intensa de luz, é adequada para plantios mistos (Inoue, 1977).

No Brasil, é freqüentemente encontrada na Floresta Ombrófila Densa Submontana (Floresta Amazônica e Floresta Atlântica), sendo restrita no Pará às "matas de terra firme". Na Região Sul do Brasil, ocorre de uma a três árvores por hectare, representando uma das estratégias encontradas pela espécie para minimizar o ataque da "broca do cedro" (Hypsipyla grandella), praga que ataca as gemas apicais (Paraná, 1979). Com relação ao estádio sucessional, o cedrorosa desenvolve-se no interior de floresta primária, porém, apresenta agressividade na vegetação secundária (Carvalho, 1994). É espécie considerada madeira de lei, de importante valor econômico, podendo atingir 20-35 m de altura, com tronco de 60-90 cm de diâmetro (Lorenzi, 1988); contudo, corre grande risco de extinção (Figliolia et al., 1986/1988).
A floração e a produção de frutos maduros no Estado de São Paulo ocorrem, respectivamente, de setembro a janeiro e de junho a setembro, com a árvore totalmente desfolhada. Cada fruto, cápsula, produz entre 30 e 100 sementes, cuja dispersão é anemocórica, possuindo uma extremidade alada, comprimida lateralmente, com até $35 \mathrm{~mm}$ de comprimento e $15 \mathrm{~mm}$ de largura, de cor bege a castanho-avermelhada e pequeno núcleo seminal. Um quilograma contém de 16.000 a 56.818 unidades (Carvalho, 1994, Heywood, 1978).

Segundo Roberts (1973), semente ortodoxa pode ser armazenada com umidade da semente inferior a $5 \%$ e a baixas temperaturas, por longo período, sem prejudicar seu potencial germinativo. Entretanto, conforme Carvalho(1994), semente de cedro, embora ortodoxa, perde gradativamente a viabilidade em condições ambientes de baixa umidade.

De acordo com Marchetti (1984), a semente armazenada em condições ambientais perdeu $20 \%$ da capacidade germinativa inicial, aos 60 dias. Porém, quando armazenada em câmara fria a baixa umidade, manteve a viabilidade integral por até três anos (ALCALAY, 1991). Semente de cedro com germinação inicial de $86 \%$, liofilizada a $4 \%$ de umidade, acondicionada em vidro hermeticamente fechado e envolto em papel alumínio e armazenada em ambiente de laboratório apresentaram germinação de $65 \%$, após 520 dias, enquanto a não liofilizada exibiu $0 \%$ (Figliolia et al. 1986/1988).

Amaral e Nakagawa (1989) afirmam que, em condições não controladas, a semente de espécies do gênero Cedrela, C. fissilis e C. odorata, apresenta baixa longevidade e, por esse motivo, recomendam a semeadura imediatamente após o amadurecimento dos frutos.

Figliolia et al. (1988) observaram que semente armazenada em ambiente frio ou seco conserva o poder germinativo (96\%) por 240 dias, mas aquelas mantidas em ambiente não controlado apresentam $34 \%$ de germinação, após o mesmo período. Os mesmos autores constataram a importância do grau de umidade inicial da semente e os resultados mais favoráveis foram observados em semente com $7,5 \%$ de água, enquanto a armazenada com $12,4 \%$ perdeu a viabilidade, rapidamente. 
Desse modo, observa-se que a umidade e a temperatura têm grande influência na conservação da semente, influenciando as reações bioquímicas que regulam o metabolismo envolvido no processo.

Os trabalhos disponíveis sobre semente de cedro-rosa (Cedrela fissilis Vellozo), além de escassos, não permitem o estabelecimento de tecnologias de armazenamento alicerçadas no conhecimento científico existente. Dessa maneira, considerando a importância da produção de mudas para reflorestamento, há a necessidade de aprofundamento da busca de informações sobre o tema.

O objetivo desta pesquisa foi estudar o comportamento fisiológico da semente de cedro-rosa (Cedrela fissilis Vellozo) por meio de variações no grau de umidade da semente e na temperatura do ambiente, durante o armazenamento.

\section{MATERIAL E MÉTODOS}

A pesquisa foi realizada no Laboratório Central de Sementes e Mudas do Departamento de Sementes, Mudas e Matrizes (LCSM/DSMM) da Coordenadoria de Assistência Técnica Integral (CATI), em Campinas. A semente foi proveniente do Núcleo de Produção de Mudas de Pederneiras (DSMM/CATI).

Os frutos foram coletados maduros, porém ainda fechados, de quatro plantas matrizes e colocados em ambiente sombreado, para posterior extração da semente. Foi determinado o grau de umidade inicial do lote (Brasil, 1992) e, paralelamente, obtida a amostra representativa do maior teor de água desejado $( \pm 16$ \%). Em seguida, a semente remanescente foi submetida à secagem em secador com circulação constante de ar a $30^{\circ} \pm$ $3^{\circ} \mathrm{C}$, para a obtenção dos demais graus de umidade desejados $(12,0 \%, 8,0 \%$ e $4,0 \%)$.

Os teores de água foram obtidos com acompanhamento da perda de peso da semente, durante a secagem. As amostras de semente para esse monitoramento, com pesos iniciais previamente conhecidos, foram acondicionadas em sacos de filó e distribuídas nas bandejas do secador para pesagens a intervalos regulares. Os pesos finais das amostras, correspondentes a cada um dos graus de umidade desejados, foram calculados por meio da equação descrita por Cromarty et al. (1985):

$\mathrm{Pf}=\mathrm{Pi}(100-\mathrm{Ui})(100-\mathrm{Uf})^{-1}$, onde:

$\mathrm{Pf}=$ peso da amostra $(\mathrm{g})$ após a secagem;

$\mathrm{Pi}=$ peso da amostra $(\mathrm{g})$ antes da secagem;

$\mathrm{Ui}=$ grau de umidade $(\%)$ antes da secagem;

$\mathrm{Uf}=$ grau de umidade $(\%)$ desejado após a secagem.

À medida que os graus de umidade estavam próximos dos desejados, as amostras foram retiradas, homogeneizadas e divididas em frações que, por sua vez, foram embaladas em sacos de polietileno de $0,14 \mathrm{~mm}$ de espessura e mantidas a $20^{\circ} \pm 2^{\circ} \mathrm{C}$, provisoriamente, até a obtenção de todos os teores de água.

As amostras, assim embaladas, correspondentes aos diferentes graus de umidade, foram armazenadas em câmaras a $10^{\circ}$ e $20^{\circ} \mathrm{C}$, sem controle de umidade relativa do ar.

No início e após 40, 120, 200, 280 e 360 dias de armazenamento, a semente foi submetida às seguintes avaliações: Determinação do grau de umidade: foi feita a $105^{\circ} \pm 3^{\circ} \mathrm{C} / 24$ horas, pelo método da estufa (Brasil, 1992), em duas amostras de $1 \mathrm{~g}$ por repetição. Os resultados obtidos, com base no peso úmido $(\mathrm{Bu})$, foram expressos em porcentagem. Teste de germinação: foi determinado utilizando 100 sementes (cinco subamostras de 20 sementes), dentro de recipiente de vidro mantidos a $25^{\circ} \mathrm{C}$ e fotoperíodo de 8 horas de luz e 16 horas de escuro. O substrato foi umedecido com volume de água equivalente a três vezes o seu peso sem hidratação. As avaliações, realizadas aos 11 e 16 dias após a instalação do teste, forneceram dados que foram expressos em porcentagem de plântulas normais (Andrade e Pereira, 1994). Emergência das plântulas: foi medida semeando, individualmente, 100 sementes (cinco subamostras de 20 sementes) em caixas gerbox com areia, a $2 \mathrm{~cm}$ de profundidade, dispostas em ambiente sombreado desprovido de controles de temperatura e umidade relativa. A disponibilidade hídrica foi mantida próxima da capacidade de campo. Foram consideradas as plântulas que, após 30 dias da instalação do teste, apresentaram a parte área exposta acima da superfície do substrato. Comprimento do hipocótilo da plântula: foi verificado aos 30 dias da instalação do teste de emergência, quando foram tomadas as distâncias $(\mathrm{cm})$ da região de transição da raiz com o hipocótilo à região de inserção das folhas cotiledonares. Os dados médios foram obtidos pelo quociente entre o somatório das medidas registradas em cada determinação e o número de semente utilizada.

$\mathrm{O}$ delineamento experimental foi o inteiramente casualizado, em esquema fatorial envolvendo quatro tratamentos (graus de umidade), no início do armazenamento, e oito tratamentos (quatro graus de umidade $\mathrm{x}$ duas condições térmicas) e cinco repetições, em cada época de avaliação, durante o armazenamento. A comparação das médias foi realizada pelo Teste de Tukey, ao nível de $5 \%$.

\section{RESULTADOS E DISCUSSÃO}

Pelas avaliações realizadas no início do armazenamento (Tabela 1), há proximidade entre os valores desejados e os experimentalmente obtidos para o grau de umidade. Por motivos não detectados, a semente com o teor de água de 12,4 \% exibiu, em valores absolutos, um índice de germinação 
substancialmente menor do que os dos demais graus de umidade; porém, a análise estatística não revelou diferenças significativas de germinação entre os teores de água.

Durante o período experimental, foi observada satisfatória estabilidade dentro de cada um dos graus de umidade, em relação aos dados alcançados no início do armazenamento (Tabela 2). Desse modo, a embalagem utilizada demonstrou eficiência na manutenção da identidade dos tratamentos e permitiu confiabilidade nas comparações realizadas durante o armazenamento.

TABELA 1. Grau de umidade(U)egerminação(G) da semente de cedro, no início do armazenamento (1).

\begin{tabular}{ccc}
\hline \multicolumn{2}{c}{$\mathrm{U}(\%)$} & $\mathrm{G}(\%)$ \\
\cline { 1 - 2 } Desejado & Obtido & \\
\hline 16 & 16,3 & $78,9 \mathrm{a}$ \\
12 & 12,4 & $66,2 \mathrm{a}$ \\
8 & 8,1 & $84,3 \mathrm{a}$ \\
4 & 4,5 & $83,7 \mathrm{a}$ \\
\hline
\end{tabular}

(1) Médias seguidas de mesma letra na coluna não diferem entre si pelo teste de Tukey, a $5 \%$ de probabilidade.
Aos 40 dias, não houve efeito de níveis de umidade, temperatura e interações, com relação à germinação (Tabela

TABELA 2. Grau de umidade da semente de cedro: valores médios $(\%$, bu) obtidos durante 0 armazenamento.

\begin{tabular}{|c|c|c|c|c|c|c|}
\hline \multirow{2}{*}{$\begin{array}{c}\text { Temperatura } \\
\text { da câmara de } \\
\text { armazenamento } \\
\left({ }^{\circ} \mathrm{C}\right)\end{array}$} & \multirow{2}{*}{$\begin{array}{c}\text { Grau de } \\
\text { umidade } \\
\text { da semente } \\
\text { armazenada } \\
(\%)\end{array}$} & \multicolumn{5}{|c|}{$\begin{array}{l}\text { Período de armazenamento } \\
\text { (dias) }\end{array}$} \\
\hline & & 40 & 120 & 200 & 280 & 360 \\
\hline \multirow[t]{4}{*}{10} & 16,3 & 15,2 & 14,5 & 15,0 & 16,1 & 16,5 \\
\hline & 12,4 & 11,9 & 10,5 & 11,5 & 12,3 & 13,1 \\
\hline & 8,1 & 8,4 & 8,0 & 8,5 & 8,8 & 9,2 \\
\hline & 4,5 & 3,2 & 5,3 & 4,5 & 4,7 & 5,0 \\
\hline \multirow[t]{4}{*}{20} & 16,3 & 14,5 & 14,7 & 14,9 & 16,6 & 16,9 \\
\hline & 12,4 & 10,2 & 10,7 & 11,1 & 11,9 & 13,2 \\
\hline & 8,1 & 6,4 & 8,0 & 8,4 & 9,2 & 9,4 \\
\hline & 4,5 & 3,5 & 5,0 & 5,0 & 4,0 & 5,2 \\
\hline
\end{tabular}

TABELA 3. Germinação (\%) da semente de cedro armazenada com diferentes graus de umidade (U \%), sob diferentes temperaturas $\left(\mathrm{T}^{\circ} \mathrm{C}\right)(1)$.

\begin{tabular}{ccccccc}
\hline \multirow{2}{*}{ Fator de variação } & Tratamento & \multicolumn{5}{c}{ Período de armazenamento (dias) } \\
\cline { 3 - 6 } & & 40 & 120 & 200 & 280 & 360 \\
\hline U & 16,3 & $76,2 \mathrm{a}$ & $71,0 \mathrm{~b}$ & $68,9 \mathrm{~b}$ & $41,4 \mathrm{~b}$ & $36,9 \mathrm{c}$ \\
& 12,4 & $76,5 \mathrm{a}$ & $79,6 \mathrm{ab}$ & $91,1 \mathrm{a}$ & $78,0 \mathrm{a}$ & $79,2 \mathrm{~b}$ \\
& 8,1 & $76,7 \mathrm{a}$ & $87,0 \mathrm{a}$ & $89,1 \mathrm{a}$ & $86,3 \mathrm{a}$ & $90,0 \mathrm{a}$ \\
& 4,5 & $78,7 \mathrm{a}$ & $79,4 \mathrm{ab}$ & $83,8 \mathrm{a}$ & $84,4 \mathrm{a}$ & $84,1 \mathrm{ab}$ \\
$\mathrm{T}$ & & & & & \\
& 10 & $79,2 \mathrm{a}$ & $81,8 \mathrm{a}$ & $87,5 \mathrm{a}$ & $78,6 \mathrm{a}$ & $84,9 \mathrm{a}$ \\
& 20 & $74,9 \mathrm{a}$ & $77,2 \mathrm{~b}$ & $80,2 \mathrm{~b}$ & $68,9 \mathrm{~b}$ & $62,5 \mathrm{~b}$ \\
$\mathrm{~T}$ & & & & & \\
& $16,3 / 10$ & $77,6 \mathrm{a}$ & $66,2 \mathrm{a}$ & $78,1 \mathrm{~b}$ & $67,2 \mathrm{~b}$ & $86,1 \mathrm{a}$ \\
& $12,4 / 10$ & $77,4 \mathrm{a}$ & $75,2 \mathrm{a}$ & $96,0 \mathrm{a}$ & $81,8 \mathrm{ab}$ & $82,2 \mathrm{a}$ \\
& $8,1 / 10$ & $78,6 \mathrm{a}$ & $82,0 \mathrm{a}$ & $97,1 \mathrm{a}$ & $83,6 \mathrm{a}$ & $88,3 \mathrm{a}$ \\
& $4,5 / 10$ & $80,6 \mathrm{a}$ & $79,4 \mathrm{a}$ & $69,4 \mathrm{~b}$ & $80,0 \mathrm{ab}$ & $81,9 \mathrm{a}$ \\
& & & & & \\
& $16,3 / 20$ & $74,8 \mathrm{a}$ & $75,5 \mathrm{a}$ & $58,8 \mathrm{c}$ & $17,9 \mathrm{c}$ & $1,4 \mathrm{c}$ \\
& $12,4 / 20$ & $74,8 \mathrm{a}$ & $83,2 \mathrm{a}$ & $84,5 \mathrm{ab}$ & $73,8 \mathrm{~b}$ & $75,3 \mathrm{~b}$ \\
& $8,1 / 20$ & $72,8 \mathrm{a}$ & $90,8 \mathrm{a}$ & $76,9 \mathrm{~b}$ & $88,7 \mathrm{a}$ & $91,7 \mathrm{a}$ \\
& $4,5 / 20$ & $76,0 \mathrm{a}$ & $75,6 \mathrm{a}$ & $94,3 \mathrm{a}$ & $88,0 \mathrm{ab}$ & $86,1 \mathrm{ab}$ \\
\hline
\end{tabular}

(1) Médias seguidas de mesma letra na coluna, dentro de cada fator de variação, não diferem entre si pelo teste de Tukey, a $5 \%$ de probabilidade. 
TABELA 4. Emergência (\%) das semente de cedro armazenada com diferentes graus de umidade (U \%) sob diferentes temperaturas $\left(\mathrm{T}^{\circ} \mathrm{C}\right)(1)$.

\begin{tabular}{|c|c|c|c|c|c|c|}
\hline \multirow{2}{*}{ Fator de variação } & \multirow{2}{*}{ Tratamento } & \multicolumn{5}{|c|}{ Período de armazenamento (dias) } \\
\hline & & 40 & 120 & 200 & 280 & 360 \\
\hline \multirow[t]{4}{*}{$\mathrm{U}$} & 16,3 & $60,2 \mathrm{~b}$ & $80,4 \mathrm{a}$ & $59,0 \mathrm{~b}$ & $41,4 \mathrm{~b}$ & $36,9 \mathrm{c}$ \\
\hline & 12,4 & $70,3 \mathrm{ab}$ & 81,4 a & $81,3 \mathrm{a}$ & $78,0 \mathrm{a}$ & $79,2 \mathrm{~b}$ \\
\hline & 8,1 & $79,8 \mathrm{a}$ & $86,7 \mathrm{a}$ & $86,5 \mathrm{a}$ & 86,3 a & $90,0 \mathrm{a}$ \\
\hline & 4,5 & 80,6 a & $79,8 \mathrm{a}$ & $79,4 \mathrm{a}$ & 84,4 a & $84,1 \mathrm{ab}$ \\
\hline \multirow[t]{2}{*}{$\mathrm{T}$} & 10 & 77,9 a & 82,9 a & $82,4 \mathrm{a}$ & $78,6 \mathrm{a}$ & 84,9 a \\
\hline & 20 & $68,0 \mathrm{~b}$ & 81,4 a & $71,7 \mathrm{~b}$ & $68,9 \mathrm{~b}$ & $62,2 \mathrm{~b}$ \\
\hline \multirow[t]{8}{*}{$\mathrm{UxT}$} & $16,3 / 10$ & $68,9 \mathrm{a}$ & $84,8 \mathrm{a}$ & $70,0 \mathrm{a}$ & $67,2 \mathrm{~b}$ & $86,1 \mathrm{a}$ \\
\hline & $12,4 / 10$ & $84,5 \mathrm{a}$ & $82,6 \mathrm{a}$ & $82,4 \mathrm{a}$ & $81,8 \mathrm{ab}$ & $82,8 \mathrm{a}$ \\
\hline & $8,1 / 10$ & 78,3 a & $85,3 \mathrm{a}$ & 91,9 a & 83,7 a & 88,3 a \\
\hline & $4,5 / 10$ & $79,1 \mathrm{a}$ & $78,5 \mathrm{a}$ & $82,4 \mathrm{a}$ & $80,4 \mathrm{ab}$ & 81,9 a \\
\hline & $16,3 / 20$ & $51,2 \mathrm{~b}$ & $75,6 \mathrm{a}$ & $47,5 \mathrm{a}$ & $17,9 \mathrm{c}$ & $1,3 \mathrm{c}$ \\
\hline & $12,4 / 20$ & $53,8 \mathrm{~b}$ & $80,2 \mathrm{a}$ & 80,3 a & $73,9 \mathrm{~b}$ & $75,3 \mathrm{~b}$ \\
\hline & $8,1 / 20$ & $81,3 \mathrm{a}$ & 87,9 a & $80,0 \mathrm{a}$ & 88,7 a & 91,7 a \\
\hline & $4,5 / 20$ & 82,0 a & 80,9 a & $76,2 \mathrm{a}$ & $88,0 \mathrm{ab}$ & $86,1 \mathrm{ab}$ \\
\hline
\end{tabular}

(1) Médias seguidas de mesma letra na coluna, dentro de cada fator de variação, não diferem entre si pelo teste de Tukey, a $5 \%$ de probabilidade.

3), provavelmente devido ao período curto de armazenamento, que não permitiu variação de comportamento entre os tratamentos. O mesmo foi observado por Piña-Rodrigues e Jesus (1992), em semente de cedro-rosa (Cedrela angustifolia S. ET. MOC), após 75 dias de armazenamento. Aos 120 dias, foram observadas algumas diferenças, tendo a semente armazenada com $16,3 \%$ de umidade, germinação inferior às demais; durante todo o período de armazenamento. Da mesma forma, a semente armazenada a $10^{\circ} \mathrm{C}$ exibiu, independente de interação, germinação superior àquelas mantidas a $20^{\circ} \mathrm{C}$, nesse período e em todos os demais. Não houve efeito de interação umidade $\mathrm{x}$ temperatura aos 40 e 120 dias, ou seja, a semente se comportou do mesmo modo, independente da temperatura. Efeitos de interação foram observados dos 200 dias, inclusive, em diante. $\mathrm{A} 10^{\circ} \mathrm{C}$, a semente mantida com quaisquer dos graus de umidade comportou-se, no geral, de modo semelhante, por todo o período de armazenamento Por outro lado, a $20^{\circ} \mathrm{C}$, a semente armazenada com teores de água entre 12,4\% e 4,5\% exibiu germinação nitidamente superior à mantida com $16,3 \%$. Figliolia (1988) observou perda considerável de viabilidade em semente de cedro-rosa (Cedrela fissilis) após 60 dias de armazenamento, com 12,4\% de água.
Tendências semelhantes foram observadas com relação à emergência e ao comprimento da parte aérea (Tabelas 4 e 5), à temperatura ambiente. Embora apresentando comportamento semelhante quando armazenada a $10^{\circ} \mathrm{C}$, a semente mantida com $16,3 \%$ de teor de água, a $20^{\circ} \mathrm{C}$, apresentou taxas de emergência e crescimento de plântula inferiores à armazenada com os demais graus de umidade, ou seja, entre $12,4 \%$ e $4,5 \%$.

Aos 280 e 360 dias, a semente mantida com $16,3 \%$ de grau de umidade, a $20^{\circ} \mathrm{C}$, se revelou imprestável para a semeadura e formação de mudas, como mostram os dados de germinação, emergência e comprimento de parte aérea (Tabelas 3, 4 e 5).

O conjunto de dados obtidos mostrou, na maioria dos casos, que os graus de umidade de entre $4,5 \%$ e $12,4 \%$ tenderam a favorecer o desempenho fisiológico da semente, nas duas temperaturas consideradas. Paralelamente, o teor de água mais elevado, ou seja, $16,3 \%$, a $20^{\circ} \mathrm{C}$, causou declínio no desempenho fisiológico, sugerindo ação prejudicial à conservação da semente. De maneira análoga, Piña-Rodrigues e Jesus (1992) constataram que o armazenamento de cedro-rosa (Cedrela angustifólia), com teor de água entre 12 e $18 \%$, pode ser crítico por períodos superiores a $60-75$ dias, quando mantidas em ambiente sem controle de temperatura e umidade relativa. 
TABELA 5. Comprimento da parte aérea (hipocótilo), em $\mathrm{cm}$, de plântula de cedro originária de semente armazenada com diferentes graus de umidade $(\mathrm{U} \%)$, sob diferentes temperaturas $\left(\mathrm{T}^{\circ} \mathrm{C}\right)(1)$.

\begin{tabular}{|c|c|c|c|c|c|c|}
\hline \multirow{2}{*}{ Fator de variação } & \multirow{2}{*}{ Tratamento } & \multicolumn{5}{|c|}{ Período de armazenamento (dias) } \\
\hline & & 40 & 120 & 200 & 280 & 360 \\
\hline \multirow[t]{4}{*}{$\mathrm{U}$} & 16,3 & $6,29 \mathrm{~b}$ & $7,87 \mathrm{bc}$ & $5,85 \mathrm{~b}$ & $1,67 \mathrm{c}$ & $2,60 \mathrm{c}$ \\
\hline & 12,4 & $7,04 \mathrm{ab}$ & $8,51 \mathrm{ab}$ & $8,68 \mathrm{a}$ & $4,65 \mathrm{~b}$ & $4,82 \mathrm{~b}$ \\
\hline & 8,1 & $7,08 \mathrm{ab}$ & $9,34 \mathrm{a}$ & $8,90 \mathrm{a}$ & $5,21 \mathrm{ab}$ & $5,63 \mathrm{ab}$ \\
\hline & 4,5 & $8,03 \mathrm{a}$ & $6,66 \mathrm{c}$ & $7,02 \mathrm{~b}$ & $5,91 \mathrm{a}$ & $6,65 \mathrm{a}$ \\
\hline \multirow[t]{2}{*}{$\mathrm{T}$} & 10 & $8,07 \mathrm{a}$ & $8,82 \mathrm{a}$ & $8,23 \mathrm{a}$ & $4,86 \mathrm{a}$ & $6,01 \mathrm{a}$ \\
\hline & 20 & $6,14 \mathrm{~b}$ & $7,37 \mathrm{~b}$ & $6,99 \mathrm{~b}$ & $3,86 \mathrm{~b}$ & $3,84 \mathrm{~b}$ \\
\hline \multirow[t]{8}{*}{$\mathrm{U} \times \mathrm{T}$} & $16,3 / 10$ & $7,90 \mathrm{a}$ & 9,49 a & $7,44 \mathrm{a}$ & $2,78 \mathrm{c}$ & $4,45 \mathrm{~b}$ \\
\hline & $12,4 / 10$ & $8,28 \mathrm{a}$ & $9,37 \mathrm{a}$ & $9,06 \mathrm{a}$ & $6,60 \mathrm{a}$ & $5,85 \mathrm{ab}$ \\
\hline & $8,1 / 10$ & $8,02 \mathrm{a}$ & $9,24 \mathrm{a}$ & $8,82 \mathrm{a}$ & $4,47 \mathrm{~b}$ & $6,64 \mathrm{a}$ \\
\hline & $4,5 / 10$ & $8,10 \mathrm{a}$ & $7,20 \mathrm{~b}$ & $7,61 \mathrm{a}$ & $5,57 \mathrm{ab}$ & 7,09 a \\
\hline & $16,3 / 20$ & $4,68 \mathrm{a}$ & $6,25 \mathrm{~b}$ & $4,26 \mathrm{c}$ & $0,56 \mathrm{c}$ & $0,75 \mathrm{c}$ \\
\hline & $12,4 / 20$ & $5,80 \mathrm{a}$ & $7,65 \mathrm{ab}$ & $8,30 \mathrm{ab}$ & $2,69 \mathrm{~b}$ & $3,79 b$ \\
\hline & $8,1 / 20$ & $6,14 \mathrm{a}$ & $9,44 \mathrm{a}$ & 8,98 a & $5,96 \mathrm{a}$ & $4,62 \mathrm{ab}$ \\
\hline & $4,5 / 20$ & $7,96 \mathrm{a}$ & $6,12 \mathrm{~b}$ & $6,44 \mathrm{~b}$ & $6,25 \mathrm{a}$ & $6,22 \mathrm{a}$ \\
\hline
\end{tabular}

(1) Médias seguidas de mesma letra na coluna, dentro de cada fator de variação, não diferem entre si pelo teste de Tukey, a $5 \%$ de probabilidade.

\section{CONCLUSÕES}

A conservação da semente de cedro, considerando o intervalo de $16,3 \%$ a $4,5 \%$ para o teor de água, é favorecida pelos graus de umidade entre $12,4 \%$ e $4,5 \%$, nas temperaturas de $20^{\circ}$ e $10^{\circ} \mathrm{C}$.

\section{AGRADECIMENTOS}

Os autores agradecem aos engenheiros agrônomos Wilson Roberto Marques Sales, do Núcleo de Produção de Mudas de Pederneiras da CATI - Sementes e Mudas, pela coleta da semente, e Antônio Carlos Silva Andrade, do Jardim Botânico do Rio de Janeiro, pelas valiosas sugestões.

\section{REFERÊNCIAS}

ALCALAY, N. Armazenamento de sementes de cedro (Cedrela fissilis Vell.). In: CONGRESSO FLORESTAL BRASILEIRO, 6., 1990, Campos do Jordão. Trabalhos voluntários - resumos. São Paulo: Sociedade Brasileira de Silvicultura, 1991. p. 88.
AMARAL, W. A. N.; NAKAGAWA, J. Dispersão, maturação e armazenamento de sementes de duas espécies do gênero Cedrela. In: SIMPÓSIO BRASILEIRO SOBRE TECNLOGIA DE SEMENTES FLORESTAIS, 2, 1989, Atibaia. Anais... São Paulo: SMA/SP e IF, 1989. p. 287.

ANDRADE, A. C. S.; PEREIRA, T. S. Efeito do substrato e da temperatura na germinação e no vigor de sementes de cedrorosa Cedrela odorata L. (MELICEAE), Revista Brasileira de Sementes, Brasília, v. 16, n. 1, p. 34-40, 1994.

AGUIAR, I. B.; PINA-RODRIGUES, F.C. M.; FIGLIOLIA, M. B. Sementes florestais. Brasília: ABRATES, 1993. 350p.

BRASIL, Ministério da Agricultura e Reforma Agrária. Regras para análise de sementes. Brasília: SNDA/DNDV/ CLAV, 1992. 365p.

CARVALHO, P. E. R. Espécies florestais brasileiras: recomendações silviculturais, potencialidades e uso da madeira. Embrapa-CNPF, Brasília, Embrapa-SPI, 1994.

CROMARTY, A. S.; ELLIS, R. H.; ROBERTS, E. H. Design of seed storage facilities for genetic conservation. Rome: Internacional Board of Plant Genetic Resources, 1985. 100p. 
FIGLIOLIA, M. B.; SILVA, A. da.; JARDIM, D. C. P.; YWANE, M. S. Viabilidade de sementes liofilizadas de essências florestais nativas. Silvicultura em São Paulo, v.20/22, p.47-55, 1986/1988.

FIGLIOLIA, M. B. Conservação de sementes de essências florestais. Boletim Técnico IF, São Paulo, v.43, p.1-18, 1988.

HEYWOOD, V. H. Flowering plants of the world. New York: Mayflower Books, 1978. 335p.

INOUE, M.T. A Auto-ecologia do gênero Cedrela: efeitos na fisiologia do crescimento no estágio juvenil em função da intensidade luminosa. Floresta, Curitiba, v.7, n.2, p.58-61, 1977.

LORENZI, H. Árvores Brasileiras: manual de identificação e cultivo de plantas arbóreas nativas do Brasil. Nova Odessa, SP: Editora Plantarum, 1998. v.1, 368p.
MARCHETTI, E. R. Época de coleta, semeadura, tratamento pré-germinativo e métodos de semeadura de espécies florestais cultivadas no Rio Grande do Sul. In: CONGRESSO FLORESTAL ESTADUAL, 5., 1984, Nova Prata. Anais... Nova Prata: Prefeitura Municipal, 1984, v.2, p.524-532.

PIÑA-RODRIGUES, F. C. M.; JESUS, R. M. Comportamento das sementes de cedro-rosa (Cedrela angustifolia S. ET. MOC) durante o armazenamento. Revista Brasileira de Sementes, Brasília, v.14, n.1, p.31-36, 1992.

PARANÁ. Universidade Federal. Centro de Pesquisas Florestais. Estudos das alternativas técnicas, econômicas e sociais para o setor florestal do Paraná; sub-programa Tecnologia. Curitiba: SUDESUL/UFPR, 1979. 335p.

ROBERTS, E. H. Predicting the storage life of seeds. Seed Science and Technology, Zurich, v. 1, p.499-514, 1973. 